\title{
Quantitative Analysis of Biomechanical Parameters in CMJ and SJ Jump Tests on 10-14 Years Old Players of Tirana Futball Club
}

\author{
Aida Bendo*, Ferdinand Mara
}

Sports University of Tirana, Faculty of Physical Activity and Recreation, Department of Health and Movement, Tirana, Albania

DOI: $10.36348 /$ jaspe.2020.v03i05.003

| Received: 16.05.2020 | Accepted: 23.05.2020 | Published: 28.05.2020

*Corresponding author: Aida Bendo

Abstract

Vertical jumping ability is of importance for good performance in different kinds of sports, and football among them. The most effective way for a football coach to improve vertical jumping ability is to enhance the maximal jump height (Hmax) and maximal force (Fmax) output in a training program, such as proprioception training. The purpose of this study is to estimate the quantitative analysis of biomechanical variables in counter movement jump (CMJ) and squat jump (SJ) tests. The participating subjects of this study were 29 boys aged 10-14 years old, players of Tirana Football Club (FC). According to the results of the t-test values, it was concluded that there was a statistical improvement of $7 \mathrm{~cm}$ in maximal height (CMJ test) and an improvement of $320 \mathrm{~N}$ in maximal force (SJ test). t-test analysis applied at this team, through the mean comparisons in three different phases of measuremets, emphasizes the differences between them and their statistical and practical significance. Two way ANOVA analysis within subjects showed a significant effect of training level, as a result of which it was concluded clearly that the jumping technique strongly affect the mechanical output muscles, as the motors that generate explosion maximal force and also maximal jump height. The proprioception exercises have a huge effect in the variety of training, by changing the style, monotony, the difficulty, which results in improving all biomechanical parameters including those of the sportive technique.

Keywords: Counter movement jump, squat jump, proprioception training.

Copyright @ 2020: This is an open-access article distributed under the terms of the Creative Commons Attribution license which permits unrestricted use, distribution, and reproduction in any medium for non-commercial use (NonCommercial, or CC-BY-NC) provided the original author and source are credited.

\section{INTRODUCTION}

One of the common features of human movement is its variability. Human movement variability can be described as normal variations, which occurs in motor performance during multiple repetitions of a task [14]. One study statet that jumping is a simple plyometric task required in a variety of athletic activities [6], while another study, had emphasis that vertical jumping ability is of importance for good performance in different kinds of sports, such as basketball, voleyball and football among them [4]. An interesting study was found the most effective way for a coach is to improve the maximal jump height (Hmax) and maximal force (Fmax) output in a program [2], such as proprioception training. More recently, vertical jump height has been used as a standard measure to assess the efficiency of the stretch-shortening cycle [8]. Some research reports have indicated that vertical jumps preceded by an eccentric contraction result in greater vertical jump heights $[1 ; 9 ; 8]$. The purpose of this study is to estimate the quantitative analysis of biomechanical variables in counter movement jump
(CMJ) and squat jump (SJ) tests. In the literature, was found that both tests are considered as wellstandardized protocols [13]. A Leonardo Mechanography force plate is used to assess ground reaction forces (GRF) during movements [10]. The most important factor to be controlled is vertical jump technique, which affect directly to the results of Hmax and Fmax in both tests. The height in takeoff is affected directly by vertical velocity at takeoff and by gravity. Based on second Newton's law, considering only vertical component, the forces that acts at a body in $\mathrm{Z}$-direction are: the reaction ground force $\mathrm{F}_{\mathrm{Z}}(\mathrm{t})$ and the body weight $\mathrm{mg}$, which are related to the vertical acceleration as follow:

$$
\sum F_{Z}=m \frac{d v_{z}}{d t}=F_{Z}(t)-m g
$$

Solving the above equation for $\frac{d v}{d t}$ and integrating the results as a time function, the velocity of the mass center in takeoff is given by expression: 


$$
v_{t_{0}}=\frac{1}{m} \int_{t_{1}}^{t_{2}}\left[F_{Z}(t)-m g\right] d t
$$

Where $t_{1}$ is a point before jump in force plate and $t_{2}$ is the takeoff time. So the jump height of the mass center is simply a function of takeoff velocity. During a vertical jump, the total impulse given to a body, will determine the jump height, which can be reachable:

$$
L_{Z}=\int_{t_{1}}^{t_{2}} F_{Z}(t) d t=m \int_{t_{1}}^{t_{2}} v_{z}(t) d t
$$

The jump height of a subject can be determined through energetically method, based on recent findings [3]. According to the energy conservation law, the kinetic energy of the mass center to the potential energy of the body mass center in the peak of the height can be expresses as below:

$$
E_{K_{t_{0}}}+E_{P_{t_{0}}}=E_{K_{h}}+E_{P_{h}}
$$

Vertical velocity at take-off can be used to determine jump height. So, from the above equation was found that:

$$
H_{j u m p}=-\left(\frac{v_{\text {take-off }}^{2}}{2 g}\right)
$$

Jumping ability may be improved in several methods, which includes: general and specific exercises, as well as the proprioception exercises. All of them develop the jumping musculature, and velocity component of power in a way, which is specific to the required technique of a subject.

\section{MATERIAL AND METHODS}

Subjects participating were 29 boys aged 1014 , mean $(12.1 \pm 0.62)$ years old and they were taken from Tirana football club team. The measurements were recorded in force plate Biomechanics Laboratory of SUT, according to Leonardo Mechonagraphy protocol
[10]. The study was approved and provided by the Sports University of Tirana. Informed consent was obtained from the parents of subjects, in order to consider them as participants of the study. The standard jumping testing protocol was used to collect the data, in two different forms: CMJ (counter movement jump) test - test for maximal height, performed with arm swing, and SJ (squat jump) test- test for maximal force, jump without arm swig with arms closed to the waist. When CMJ are compared with SJ, there is empical evidence that the average height of the jumps is greater than $10 \%$ when arms are used, based on the some studies raported [13, 7, 11, 15]. Statistical analyses were performed using SPSS version 17 . The study period includes three phases. The initial phase: before training measurements, middle: after six months and the final phase: nine months after training measurements. The program was based in propriocpetive training which included: plyometric, izokinetic and kinetic chanin exercises. During the processing of the results, statistical analysis of t-test and two way ANOVA analysis, were carried, for the quantitative analysis of all biomechanical parameters, that were subject and the focus of this study. Statistical significance was set for all statistical procedures at $\mathrm{p} \leq 0.05$.

\section{RESULTS}

Table 1 gives descriptive statistis of the anthropometric parameters of this team. Subjects participating were 29 boys aged 10-14, mean $(12.1 \pm$ $0.62)$ years old, with these anthropometric charactaristics: height $(1.54 \pm 0.14) \mathrm{m}$, body mass $(46.55 \pm 9.74) \mathrm{kg}$ and body mass index (BMI) $(19.3 \pm$ $2.37) \mathrm{kg} / \mathrm{m}^{2}$.

Table 1 generates also descriptive statistics for the variables obtained during the three measurements of

\begin{tabular}{|c|c|c|c|c|c|c|c|}
\hline \multicolumn{2}{|c|}{ Measurement } & Parameter & Mean \pm SD & Rank & $\begin{array}{l}\text { Min. } \\
\text { Value }\end{array}$ & $\begin{array}{l}\text { Max. } \\
\text { Value }\end{array}$ & Variance \\
\hline \multirow{4}{*}{\multicolumn{2}{|c|}{$\begin{array}{l}\text { Anthropometric } \\
\text { characteristics }\end{array}$}} & $\begin{array}{c}\text { Age } \\
\text { (years old) }\end{array}$ & $12.10 \pm 0.62$ & 4.00 & 10.00 & 14.00 & 0.38 \\
\hline & & Height (m) & $1.54 \pm 0.94$ & 0.40 & 1.36 & 1.76 & 0.01 \\
\hline & & Body mass (kg) & $46.55 \pm 9.74$ & 38.40 & 31.90 & 70.30 & 94.81 \\
\hline & & BMI $\left(\mathrm{kg} / \mathrm{m}^{2}\right)$ & $19.30 \pm 2.37$ & 8.71 & 15.58 & 24.29 & 5.63 \\
\hline \multirow{3}{*}{$\begin{array}{c}\text { CMJ } \\
\text { test }\end{array}$} & Phase I & $\mathrm{H} 1 \mathrm{max}(\mathrm{m})$ & $0.37 \pm 0.05$ & 0.21 & 0.27 & 0.48 & 0.003 \\
\hline & Phase II & $\mathrm{H} 2 \max (\mathrm{m})$ & $0.42 \pm 0.57$ & 1.16 & 0.33 & 0.49 & 0.003 \\
\hline & Phase III & $\mathrm{H} 3 \max (\mathrm{m})$ & $0.45 \pm 0.05$ & 1.21 & 0.35 & 0.56 & 0.003 \\
\hline \multirow{3}{*}{ SJ test } & Phase I & F1max $(\mathrm{kN})$ & $1.07 \pm 0.25$ & 0.97 & 0.69 & 1.66 & 0.06 \\
\hline & Phase II & $\mathrm{F} 2 \max (\mathrm{kN})$ & $1.19 \pm 0.27$ & 1.16 & 0.81 & 1.97 & 0.07 \\
\hline & Phase III & F3max $(\mathrm{kN})$ & $1.40 \pm 0.28$ & 1.15 & 0.97 & 2.12 & 0.08 \\
\hline
\end{tabular}
both CMJ and SJ tests. The results obtained from these measurements are used to describe their characteristics.

Table-1: Statistical descriptive of anthropometric parameters and three measurements of CMJ and SJ tests

t-test analysis applied at this team, through the mean comparisons in three different phases of measuremets, emphasizes the differences between them and their statistical and practical significance. 
Table 2 gives the t-test results. This information is related to the differences between the three weighting measurements, based on the average values of the biomechanical parameters in both CMJ and SJ tests and if these differences are statistically and practically significant.
By comparing the average values between pairs of variables of vertical jump, based on the values of which is reported below: the main variable of first the CMJ test is the maximum height (Hmax), and the main variable of the second $\mathrm{SJ}$ test is the maximum force (Fmax).

Table-2: Pair comparisons of the same parameters within the CMJ and SJ tests.

\begin{tabular}{|c|c|c|c|c|c|c|}
\hline \multirow[t]{2}{*}{ Parameter } & \multirow{2}{*}{$\begin{array}{c}\text { Pairs of } \\
\text { parameters }\end{array}$} & \multirow{2}{*}{$\begin{array}{c}\text { Mean Differences } \\
\text { 土SD }\end{array}$} & \multicolumn{2}{|c|}{ 95\% CI of difference } & \multirow{2}{*}{$\begin{array}{l}\text { t-test } \\
\text { value }\end{array}$} & \multirow{2}{*}{$\begin{array}{l}p- \\
\text { value }\end{array}$} \\
\hline & & & $\begin{array}{c}\text { The lower } \\
\text { limit }\end{array}$ & $\begin{array}{c}\text { The upper } \\
\text { limit }\end{array}$ & & \\
\hline \multirow{3}{*}{$\begin{array}{l}\operatorname{Hmax}(\mathrm{m}) \\
\text { in CMJ test }\end{array}$} & $\mathrm{H} 1-\mathrm{H} 2$ & $-0.04 \pm 0.03$ & -0.058 & -0.034 & -8.058 & 0.000 \\
\hline & $\mathrm{H} 2-\mathrm{H} 3$ & $-0.03 \pm 0.02$ & -0.04 & -0.02 & -7.547 & 0.000 \\
\hline & $\mathrm{H} 1-\mathrm{H} 3$ & $-0.07 \pm 0.03$ & -0.09 & -0.06 & -11.050 & 0.000 \\
\hline \multirow{3}{*}{$\begin{array}{c}\begin{array}{c}\text { Fmax } \\
(\mathbf{k N})\end{array} \\
\text { in SJ test }\end{array}$} & $\mathrm{F} 1-\mathrm{F} 2$ & $-0.12 \pm 0.09$ & -0.15 & -0.08 & -6.735 & 0.000 \\
\hline & $\mathrm{F} 2-\mathrm{F} 3$ & $-0.21 \pm 0.20$ & -0.03 & -0.13 & -5.584 & 0.000 \\
\hline & F1 - F3 & $-0.32 \pm 0.22$ & -0.41 & -0.24 & -8.014 & 0.000 \\
\hline
\end{tabular}

\section{DISCUSSION}

The first test CMJ is a test for the maximum height with the variables taken during different phases of training. In function of the main parameter the maximum height, are studied for all the other parameters of the vertical jump.

Table 2 provides the paired t-test results. This information is related to the differences between the three weighting measurements, based on average measurement of the biomechanical variables in the first CMJ test, in the second SJ test, and if these differences are statistically and practically significant.

To make the maximal height estaimation, some measurements have been made, by comparing the average values between pairs of variables of vertical jump, based on the values of which is reported below:

The primary variable of the CMJ test is the maximum height (Hmax), as it is shown in fig.1. The results obtained by the table 1 for $\mathrm{H} 1 \max (0.37 \pm 0.05)$ and $\mathrm{H} 2 \max (0.42 \pm 0.05)$ show an improvement on average of $(0.04 \pm 0.03)$, calculated with $11.9 \%$, for $\mathrm{H} 2 \max$ and $\mathrm{H} 3 \max (0.45 \pm 0.05)$ this improvement is further on average $(0.03 \pm 0.03)$, or $6.6 \%$, but a little smaller, compare the first case. By measuring H1max in $\mathrm{H} 3 \max (0.45 \pm 0.05)$ there is a very good improvement on average of $(0.07 \pm 0.03)$, calculated at $17.7 \%$. Table 2 shows that $\mathrm{t}$-test statistic values are respectively: $\mathrm{t}(28)$ $=-8.058 ; \mathrm{t}(28)=-7.547 ; \mathrm{t}(28)=-11.050, \mathrm{p}<0.005$, which confirm the statistical significance of these differences.

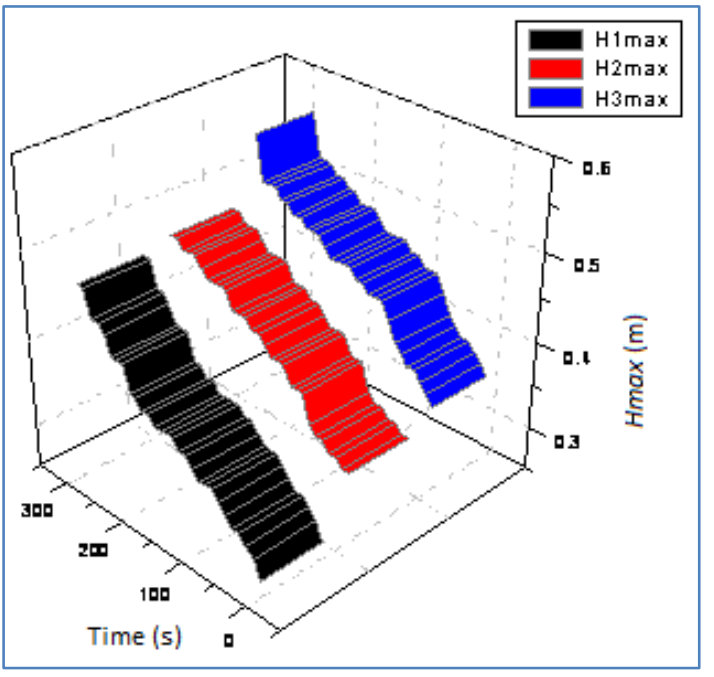

Fig-1: Hmax dependence chart from the test time for all subjects of Tirana FC team for the three CMJ test measurements

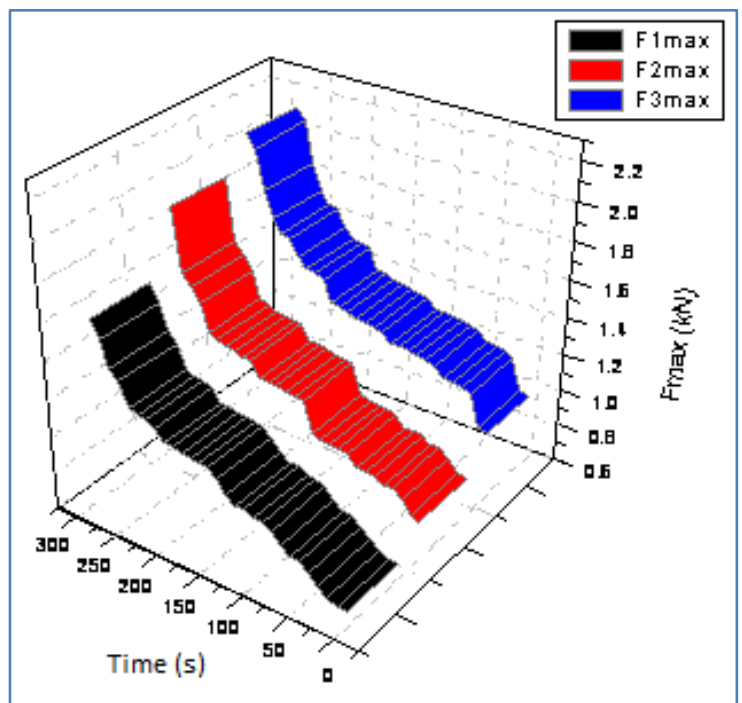

Fig-2: Maximum strength force (Fmax) variance chart from test time for all subjects of the Tirana FC team, for the three SJ test measurements 
The second SJ test is the test for the maximum force output with the variables taken during three different stages of training. Figure 2 presents Fmax. parameter, for three SJ test measurements. In function of the main parameter, the maximum force, are studied for all the other parameters of the vertical jump.

The evaluation of the results obtained from table 1 for F1max $(1.07 \pm 0.25)$ and F2max $(1.19 \pm$ $0.27)$, has yielded an improvement on average of $(0.12$ \pm 0.09 ), calculated by $10.1 \%$, for $\mathrm{F} 2 \max$ and $\mathrm{F} 3 \mathrm{max}$
$(1.40 \pm 0.28)$, there is a further improvement on average by $(0.21 \pm 0.20)$, or $15 \%$, which is greater compared to the first case, while for për F1max and F3max there is a very good improvement on average of $(0.32 \pm 0.22)$, calculated at $23.6 \%$. the t-test statistics values according to table 2 , are respectively: $\mathrm{t}(28)=-6.735 ; \mathrm{t}(28)=-$ $5.584 ; \mathrm{t}(28)=-8.014, \mathrm{p}<0.005$, which confirm that these results are statstically significant.

The Hmax parameter is clearly the highest value in CMJ test, as seen in figure 3.

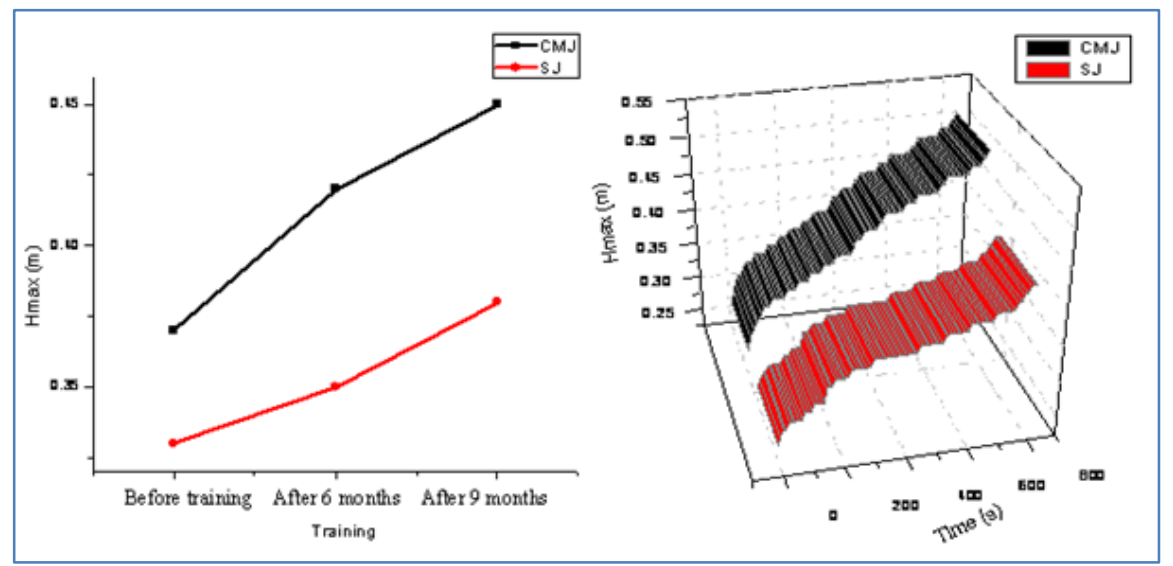

Bendo - Fig-3: 2D \& 3D graph of Hmax. parameter and training phases of "Tirana" team subjects in two vertical jump tests CMJ \& SJ

Due to the means of measurement and the direction of the t-value, it was concluded that was a statistical improvement in jump ability following the proprioception training program. In CMJ test was observed an improvement of $7 \mathrm{~cm}$, which corresponds with the results reported from one previously study [12], $(\mathrm{p}<0.001)$, whiles in SJ test was found an improvement of $320 \mathrm{~N}$ ( $\mathrm{p}<0.001)$, statistically significant both of them. In two-way ANOVA analysis within subjects, was adapted the regression model, based on Field study [5], to connect the dependent variable $y$ with the two independent variables: training and test, whilst including the interaction term, which represents the combined effect of training and test, which can be expressed as following:

$$
y_{\text {max }_{i}}=\left(b_{0}+b_{1} \text { training }+b_{2} \text { test }+b_{3} \text { interaction }\right)+\varepsilon_{i}
$$

From the comparison of Hmax variable in both of vertical jump tests CMJ and SJ, the results showed that there was not a significant effect of interaction term: training*test in Hmax mean, but there was a simple main effect of training level $(\mathrm{p}<0.001)$ and a significant effect of type of test term $(\mathrm{p}<0.001)$.

Regarding the parameter of Fmax, it dominates in the $\mathrm{Sj}$ test, and this is shown in figure 4.

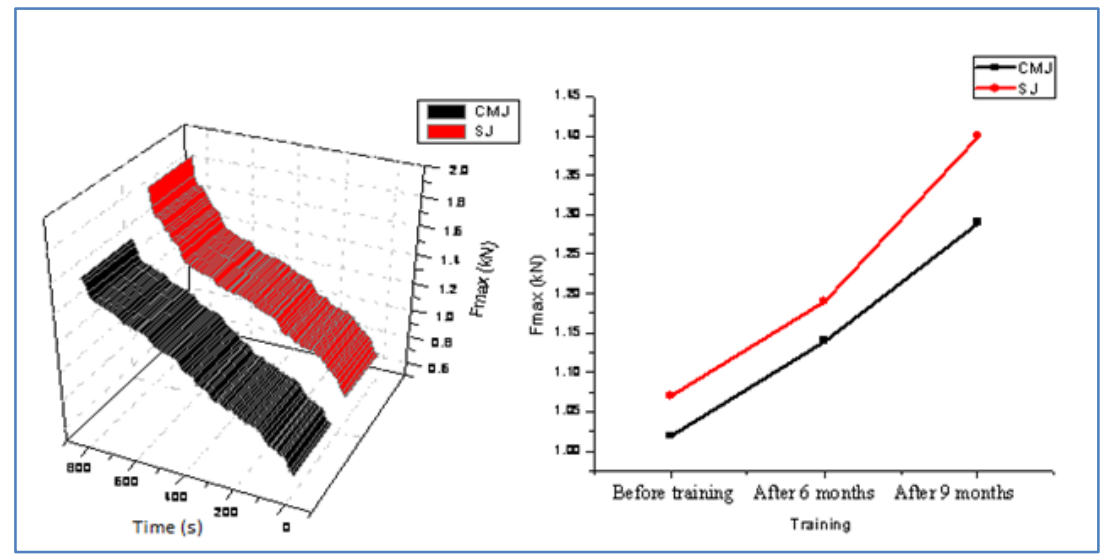

Fig-4: 2D \& 3D graph of Fmax. Parameter and training phases of "Tirana" team subjects in two vertical jump tests CMJ \& SJ. 
Based on the results of the variance analysis ANOVA, by the comparison of Fmax variable in both of vertical jump tests (CMJ and $\mathrm{SJ}$ ) it is not observed a significant effect of type of test term $(p>0.050)$ and no significant effect in Fmax of interaction term: training*test, but there is a significant effect of training level $(\mathrm{p}<0.001)$.

\section{CONCLUSIONS}

According to the results of the t-test values of parameters have changed as follow: in CMJ test, Hmax - an improvement of $7 \mathrm{~cm}$ and in SJ test, Fmax - an improvement of $320 \mathrm{~N}$, statistically significant both of them. Multiple equations of regression emerged these parameters as the best predictors of the main variables for each test: in CMJ test for the measurement of Hmax: the best predictor is: Vmax, while in SJ test for the measurement of Fmax the best predictors are: body mass and Pmax $/ \mathrm{kg}$. Two way ANOVA analysis within subjects showed for an term significant, effect of training level and a very important effect of the combination term: the training for the both vertical jumping tests, as a result of which we come with a conclusion that: the proprioceptive training influences in a clear way in the improvement of parameters results, Hmax in both vertical jumping tests (increase of average Hmax values), and Fmax in both vertical jumping tests (increase of average Fmax values). Combined general and specific proprioception training is effective as power training for improving maximal jump height and maximal output in both of tests. The proprioceptive exercises have a huge effect in the variety of trainings, by changing the style, monotony and the difficulty of training. They develop and improve the psychophysical, coordinative and locomotive condition, enabling the subject to both mentally mobilize and having a higher muscular tonification, by gradually increasing the moving efficiency, and the performance improvement. Clearly, the jumping technique strongly affects the mechanical output muscles, as the motors that generate explosion maximal force and also maximal jump height. Finally, all the proprioceptive applied exercises in Tirana FC players have influenced in obvious improvements of all biomechanical parameters, including the sportive technique. Future training exercises studies are needed to determine which technique should be preferred to the specific required tasks. This needs a close cooperation between researches and coaches in the future.

\section{REFERENCES}

1. Asmussen E, Bonde- Petersen F. Storage of elastic energy in skeletal muscles in man. Acta Physiologica Scandinavica. 1974 Jul;91(3):385-92.
2. Baker, D. (1996). Improving vertical jump performance through general, special, and specific strength training. Journal of strength and Conditioning Research, 10, 131-136.

3. Bendo, A.(2015). Effect of proprioception traing in biomechanical parameters. Disertation thesis:8-10. https://www.upt.al/images/stories/menu/Diseracion i\%20Aida\%20Bendo.pdf

4. Bobbert, M.F.(1990). Drop jumping as a training method for jumping ability. Sports Med, 9(1): 7-22

5. Field, A. (2009). Discovering statistics using spss. 2009. 3rd edition.

6. Gulick, D. T., Fagnani, J., Long, M., Morris, K., Hartzell, B., \& Epler, M. (2008). Parameters that influence vertical jump height. The Sport Journal, 11(3).

7. Harman, E. A., Rosenstein, M. T., Frykman, P. N., \& ROSenStein, R. M. (1990). The effects of arms and countermovement on vertical jumping. Med Sci Sports Exerc, 22(6), 825-833.

8. DeStaso, J., Kaminski, T. W., \& Perrin, D. H. (1997). Relationship between drop vertical jump heights and isokinetic measures utilizing the stretch-shortening cycle. Isokinetics and Exercise Science, 6(3), 175-179.

9. Komi, P. V., \& Bosco, C. (1978). Muscles by men and women. Med Sci Sport, 10, 261-5.

10. Leonardo Mechanograpy Ground Reaction Force Platform (GRFP) v4.2. St. Ed. 2010

11. Luhtanen, P., \& Komi, P. V. (1979). Mechanical power and segmental contribution to force impulses in long jump take-off. European journal of applied physiology and occupational physiology, 41(4), 267-274.

12. Mackala, K., Stodólka, J., Siemienski, A., \& Coh, M. (2013). Biomechanical analysis of squat jump and countermovement jump from varying starting positions. The Journal of Strength \& Conditioning Research, 27(10), 2650-2661.

13. Acero, R. M., Sánchez, J. A., \& Fernández-delOlmo, M. (2012). Tests of vertical jump: Countermovement jump with arm swing and reaction jump with arm swing. Strength \& Conditioning Journal, 34(6), 87-93.

14. Stergiou, N., Harbourne, R. T., \& Cavanaugh, J. T. (2006). Optimal movement variability: a new theoretical perspective for neurologic physical therapy. Journal of Neurologic Physical Therapy, 30(3), 120-129.

15. Shetty, A. B., \& Etnyre, B. R. (1989). Contribution of arm movement to the force components of a maximum vertical jump. Journal of Orthopaedic \& Sports Physical Therapy, 11(5), 198-201. 